\title{
Prolonged pruritic rash following influenza A (H1N1) vaccination
}

\author{
Yan $\underline{\text { in }}^{1}$, MRCP, MMed, Colleen Kim $\underline{\text { Thomas }}^{1}$, MRCP, FRCP
}

\begin{abstract}
In Singapore, the approved influenza A (H1N1) vaccines are Panvax ${ }^{\circledR}$ and Pandemrix ${ }^{\circledR}$. An estimated 425,000 doses of Panvax and less than 100 doses of Pandemrix had been distributed in Singapore from November 2009 to February 2010. Reviews on the H1N1 vaccine have concluded that it has a safety profile similar to that of seasonal influenza vaccines. From the time the H1N1 vaccination was implemented in Singapore on November 3, 2009, up to October 11, 2010, the Health Sciences Authority had received 173 adverse event reports from healthcare professionals. We report a case of prolonged illness after H1N1 vaccination.
\end{abstract}

Keywords: adverse effect, H1N1 vaccine

\section{INTRODUCTION}

The first influenza pandemic in 41 years was declared by the World Health Organization (WHO) on June 11, 2009. ${ }^{(1)}$ A novel 2009 influenza A (H1N1) virus was responsible for this pandemic. Shortly after the identification of the 2009 H1N1 virus, influenza vaccine manufacturers, together with public health and regulatory agencies, started developing a $2009 \mathrm{H} 1 \mathrm{~N} 1$ vaccine. ${ }^{(2)}$ Clinical trials that examined the immunogenicity, safety and tolerability of the H1N1 vaccine were conducted in healthy adults in Australia around July 2009. All adverse effects, including local (injection site) reactions and systemic symptoms (headache, myalgia and malaise), were reported to be of mild to moderate intensity and self-limiting. ${ }^{(3)}$ We present a case of prolonged illness in a patient after receiving the $\mathrm{H} 1 \mathrm{~N} 1$ vaccine.

\section{CASE REPORT}

A 31-year-old Chinese woman with a history of appendisectomy was administered a H1N1 vaccine (Panvax ${ }^{\circledR}$; CSL Biotherapies, Parkville, Victoria, Australia) on January 22, 2010. Two days after vaccination, she developed a fever and a generalised, itchy and painful rash. She subsequently developed a sore throat on January 28, 2010. The patient then presented to a general practitioner, who prescribed her with antibiotics. She had no history of recent travel or contact with any sick persons, nor did she have any known drug or food allergy.

The patient presented to the Singapore General Hospital on February 3, 2010 with persistent itchy rash and paresthesia (pins and needles sensation), associated with arthralgia and myalgia. Physical examination revealed periorbital swelling, linear streaks of petechiae on the legs (Fig. 1), purpura patches on the thigh (Fig. 2), faint and non-palpable erythema on the arm and abdomen (Fig. 3), and slightly injected tonsils. A diagnosis of exanthem caused by the H1N1 vaccine was made, and the patient was started on oral prednisolone and antihistamines, as well as

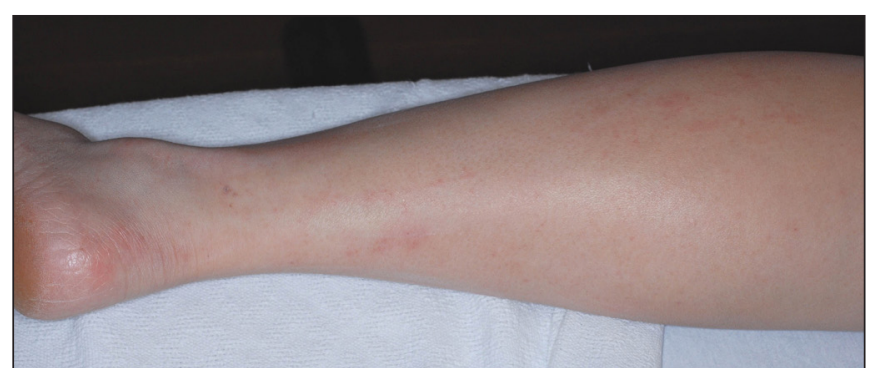

Fig. 1 Photograph shows linear streaks of petechiae on the patient's leg.

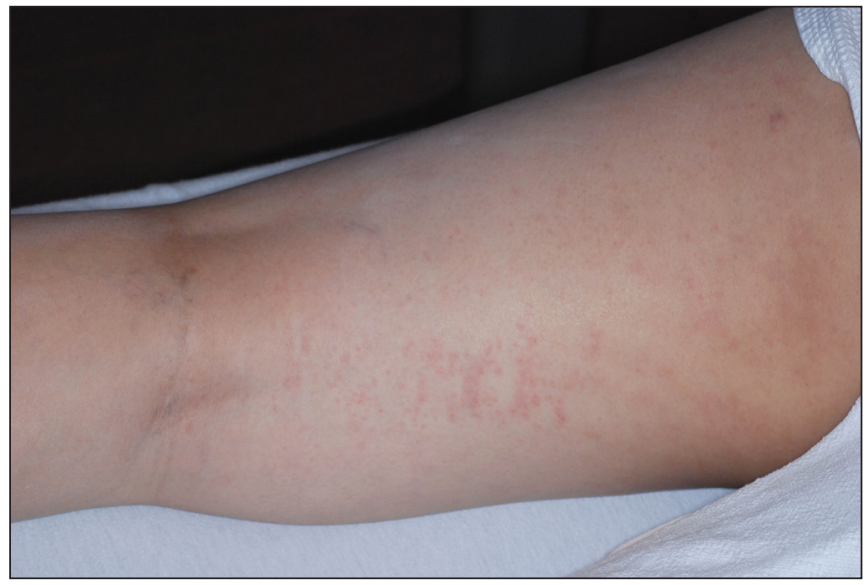

Fig. 2 Photograph shows purpura patches on the patient's thigh.

topical betamethasone cream and emollients. The patient was also given gabapentin for her paresthesia. She had low-grade fever during her five-day stay in the hospital, and oral augmentin was administered for one week. At the time of her discharge, the body rash and arthralgia had improved.

At the subsequent outpatient follow-up, the patient continued to have recurrent pruritic rash; hence, prednisolone with a tapering-down dose was continued for almost four weeks. Investigation did not reveal any underlying autoimmune or connective tissue disease. The patient made a slow recovery with minimal symptoms until her review in September 2010.

${ }^{1}$ Department of Internal Medicine, Singapore General Hospital, Singapore

Correspondence: Dr Qin Yan, Associate Consultant, Department of Internal Medicine, Singapore General Hospital, Outram Road, Singapore169608. qin_yan@yahoo.com 

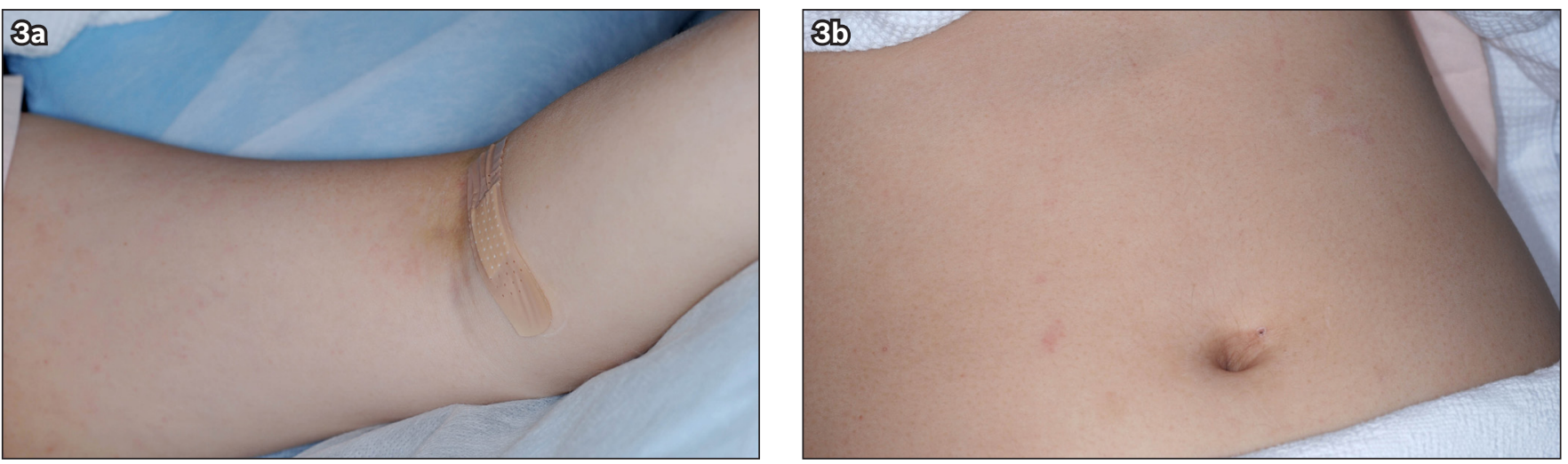

Fig. 3 Photographs show faint and non-palpable erythema on the patient's (a) arm and (b) abdomen.

\section{DISCUSSION}

In Singapore, the approved H1N1 vaccines are Panvax ${ }^{\circledR}$ and Pandemrix ${ }^{\circledR}$. An estimated 425,000 doses of Panvax and less than 100 doses of Pandemrix had been distributed in Singapore from November 2009 to February 2010.(4) The H1N1 vaccine is a monovalent, unadjuvanted, inactivated and split-virus vaccine. It is prepared in embryonated chicken eggs, employing the same standard techniques used for the production of seasonal trivalent inactivated vaccine. ${ }^{(3)}$

When assessing the safety of a vaccine, it is important to be aware of the background rates of diseases in the population, in order to separate legitimate safety concerns from events that are temporarily associated with, but not caused by, the vaccine.(5) To date, the pandemic H1N1 vaccine appears to be safe and well tolerated. Since the pandemic H1N1 vaccine is similar in design to the seasonal influenza vaccine, adverse effects are expected to be similar to those seen following seasonal influenza vaccination. The United States (US) Center for Disease Control and Prevention (CDC) reported the vaccine safety results for the pandemic $\mathrm{H} 1 \mathrm{~N} 1$ vaccines for the first two months of the vaccination campaign (October 1 to November 24, 2009), based on 3,783 reports from the US Vaccine Adverse Event Report System (VAERS) and data from 438,376 individuals vaccinated in managed-care organisations, through the Vaccine Safety Datalink (VSD). ${ }^{(6)}$ The VAERS data reported 82 adverse events per million pandemic H1N1 vaccine doses distributed, whereas that for the seasonal influenza vaccine was 47 per million doses distributed. However, no substantial difference in the proportion or types of serious adverse events was observed between the pandemic $\mathrm{H} 1 \mathrm{~N} 1$ and seasonal influenza vaccines. ${ }^{(6)}$

The Health Sciences Authority (HSA) of Singapore received 152 reported adverse events for the 425,000 doses of H1N1 vaccination distributed in Singapore from November 2009 to February 2010. ${ }^{(4)}$ The reported rate of adverse events in Singapore appears to be higher than that reported in the US. One reason for this disparity could be the higher rate of adverse events among Asian populations as compared to Caucasian populations. ${ }^{(6)}$ Notably, China reported an adverse event rate of 90 per million doses, ${ }^{(6)}$ which is also slightly higher than that reported in the US. The second possible reason for the disparity could be the variance in the reporting system of different countries. As
Singapore has a highly efficient reporting system, the adverse event rate may be more accurately represented in Singapore than in other reporting systems, i.e. the VAERS and China CDC, which may have underestimated figures due to the relatively large population size in these countries.

Clinical trials of the Panvax H1N1 vaccine conducted in healthy adults and children have not shown any occurrence of death, serious adverse events or adverse events of special interest (e.g. Guillain-Barre syndrome and immune system disorder). The most common solicited local event reported was injection-site tenderness and pain, and the most commonly reported solicited systemic events were headache, malaise and myalgia. The side-effect profile of the H1N1 vaccine, particularly the frequency and severity of solicited and unsolicited adverse events, is consistent with previous experience with seasonal influenza vaccines in adults. ${ }^{(8)}$ Among the 173 cases of adverse events reported to the HSA (as of October 11, 2011), 34 were reported as a generalised rash, of which 19 were pruritic rash. (4) Careful review of the Panvax product brochure revealed that generalised rash was not reported in healthy adults and children recruited in its clinical trials. Post-marketing surveillance revealed that pruritus, urticaria and rash were uncommon (i.e. $\geq 1 / 1,000$ and $<1 / 100$ ). ${ }^{(9)}$ This is consistent with our local data.

The case that we have reported is likely to be an extremely rare reaction to the Panvax H1N1 vaccine. Further studies are required to standardise the treatment for this type of adverse reaction.

\section{ACKNOWLEDGEMENTS}

Statistical information was adapted from the CDC and HSA websites.

\section{REFERENCES}

1. World Health Organization. DG Statement following the meeting of the Emergency Committee. In: World Health Organization: Global Alert and Response (GAR) [online]. Available at: www.who.int/csr/disease/ swineflu/4th_meeting_ihr/en/index.html. Accessed October 10, 2010.

2. Kuehn BM. CDC names H1N1 vaccine priority groups. JAMA 2009; 302:1157-8.

3. Greenberg ME, Lai MH, Hartel GF, et al. Response to a monovalent 2009 influenza A (H1N1) vaccine. N Engl J Med 2009; 361:2405-13.

4. Health Sciences Authority, Singapore. Update on safety monitoring of H1N1 vaccines from 3 November 2009 to 28 February 2010 [online]. Available at: www.hpp.moh.gov.sg. Accessed October 11, 2010. 
5. Black, S, Eskola, J, Siegrist, CA, et al. Importance of background rates of disease in assessment of vaccine safety during mass immunisation with pandemic H1N1 influenza vaccines. Lancet 2009; 374:2115-22.

6. Centres for Disease Control and Prevention (CDC). Safety of influenza A (H1N1) 2009 monovalent vaccines - United States, October 1-November 24, 2009. MMWR Morb Mortal Wkly Rep 2009; 58:1351-6.

7. Liang XF, Li L, Liu DW, et al. Safety of influenza A (H1N1) vaccine in postmarketing surveillance in China. N Engl J Med 2011; 364:638-47.

8. Talbot HK, Keitel W, Cate TR, et al. Immunogenicity, safety and consistency of new trivalent inactivated influenza vaccine. Vaccine 2008; 26:4057-61.

9. CSL Limited. Panvax ${ }^{\circledR}$ Product Information. Available at: http:// www.peelregion.ca/health/professionals/pdfs/panvax-vaccine.pdf. Accessed October 11, 2010. 